\title{
DOES ENDURANCE FATIGUE INCREASE THE RISK OF INJURY WHEN PERFORMING DROP JUMPS?
}

\author{
Kieran A. Moran ${ }^{1}$, Michelle Clarke ${ }^{1}$,Frank Reilly ${ }^{1}$, Eric S. Wallace ${ }^{2}$, Dermot Brabazon ${ }^{3}$, \\ and Brendan Marshall ${ }^{1}$ \\ 1 School of Health and Human Performance, Dublin City University, Dublin, UK; \\ 2 Sport and Exercise Sciences Research Institute, University of Ulster, Newtownabbey, UK; and \\ 3 School of Mechanical and Manufacturing Engineering, Dublin City University, Dublin, UK.
}

\begin{abstract}
Moran, KA, Clarke, M, Reilley, F, Wallace, ES, Brabazon, D, and Marshall, B. Does Endurance Fatigue Increase the risk of injury when performing drop jumps? J Strength Cond Res 23(5): 1448-1455, 2009-Although from an athletic performance perspective it may be beneficial to undertake drop jump training when fatigued (principle of "specificity" of training), such endur-ance fatigue may expose the body to a greater risk of injury if it causes an increase in peak impact accelerations. This study aimed to determine if endurance fatigue resulted in an increase in tibial peak impact acceleration and an associated change in knee kinematics when completing plyometric drop jumps. Fifteen females performed drop jumps from 3 heights $(15,30$, and $45 \mathrm{~cm})$ when fatigued and nonfatigued. Treadmill running was used to induce endurance fatigue. The following variables were assessed: tibial peak impact acceleration, knee angle at initial ground contact, maximum angle of flexion, range of flexion, and peak knee angular velocity. Fatigue resulted in significantly greater $(p<0.05)$ tibial peak impact acceleration and knee flexion peak angular velocity in drop jumps from 15 and $30 \mathrm{~cm}$, but not from $45 \mathrm{~cm}$. Fatigue had no effect on any of the knee angles assessed. The neuromuscular system was affected negatively by endurance fatigue at 15 and $30 \mathrm{~cm}$, indicating that coaches should be aware of a potential increased risk of injury in performing drop jumps when fatigued. Because from the greater drop height of $45 \mathrm{~cm}$ the neuromuscular system had a reduced capacity to attenuate the impact accelerations per se, whether nonfatigued or fatigued, this would suggest that this height may have been too great for the athletes examined.
\end{abstract}

KEY WORDS injury, plyometric, impact, landing.

\section{INTRODCUTION}

Plyometric drop jumps (DJs) are frequently used by athletes as a means of developing lower body power $(7,30,31)$. Although it is often recommended that DJs be performed when the neuromuscular system is not fatigued (31), thereby maximizing neural activation, the principle of specificity dictates that there is a clear justification for athletes who compete under fatigued conditions to develop lower limb power under such fatigued conditions (7) using a variety of training exercises, including DJs. In fact, plyometric exercises, including DJs, have been shown to enhance performance in endurance running $(29,32,34)$. However, 
performing DJs from inappropriately large heights or when fatigued may predispose athletes to lower limb injuries associated with high impact loads and accelerations $(7,22)$. In reaction to the foot contacting the ground during landing, a reactionary force causes a transient acceleration ("shock wave"), which travels up the musculo-skeletal system from the foot to the head $(4,13,21,22)$. When these impact accelerations and loads are excessive relative to the strength of the tissue, they may cause a number of musculoskeletal overuse injuries, including stress fractures $(18,39)$, osteoarthritis $(26,36,38)$, and articular cartilage and joint degeneration $(6,27,36)$. Given that the body attempts to attenuate such impact accelerations using various neuro-muscular and joint kinematic control strategies $(8,25)$, there is a potential to increase the risk of injury if DJs are completed when the neuromuscular system is fatigued $(15,16,22)$. Indeed, increased impact acceleration measured on the tibia has been observed extensively in fatigued running with associated changes in knee kinematics during landing $(5,21)$.

Only one study to date appears to have examined the effect of endurance fatigue on the magnitude of impact acceler-ations when performing DJs (from 30 and $50 \mathrm{~cm}$ ) (22). The authors concluded that fatigue had a negative effect on impact accelerations although tibial impact acceleration were only greater from $30 \mathrm{~cm}$, but not from $50 \mathrm{~cm}$. They argued that the neuromuscular system was not capable of effectively controlling the increased impact accelerations from the higher drop height $(50 \mathrm{~cm})$, and therefore when inhibited as a result of fatigue, no further significant detriment in control was evident.

Although their conclusion is tenable, it was based on results from only 2 drop heights. Clearly there is a need to examine the effect of endurance fatigue on impact accelerations from a greater number of drop heights. In addition, given that the only study to date has examined this effect in males, it would be beneficial to increase the generalization of the results by examining the effect in females. The main aim of the present study was to determine if impact acceleration, measured at the proximal tibia during DJs from increasing heights, was significantly affected by endurance fatigue. A secondary aim was to investigate if the action of the knee during the initial contact and landing phase was also affected by endurance fatigue. It was hypothesized that endurance fatigue would result in greater impact accelerations at low(er) drop heights, but not at high(er) drop heights.

\section{METHODS}

\section{Experimental Approach to the Problem}

To examine if endurance fatigue affected either the impact accelerations applied to the body or the knee kinematics used by the body, DJs were completed by all participants under nonfatigued and fatigued conditions during a single session. Three drop heights were selected. Although a number of methods have been proposed to identify an athlete's optimal drop height for training $(2,11,40)$, no studies have shown the effectiveness of one method over another for improving leg power. Therefore, the drop heights selected were based on previous DJ studies $(1,22)$ and training literature (7). Running was used to induce endurance fatigue, rather than resistance training-based exercises, because it is perhaps the most common means by which athletes become fatigued in sport. 


\section{PARTICIPANTS}

Fifteen female soccer players (age, $20.9 \pm 1.1$ years; mass, $63.4 \pm 4.9 \mathrm{~kg}$; height, $1.66 \pm 0.10$ $\mathrm{m})$ were recruited. All participants had been playing competitive soccer for at least the last 3 years and currently trained a minimum of 4 times per week during the competitive season. Throughout their season, participants undertook at least 2 gym-based resistance training session per week and testing was completed between 5 and 6 weeks into their competition season. The study inclusion criteria required participants to have at least 6 weeks of prior experience in the last 2 years in performing DJs as a means oftraining. Written informed consent was obtained in accordance with the university's ethical committee's guide-lines. Participants were excluded from the study if they had any history of lower extremity injury. Two weeks before the experimental trial, participants were demonstrated the DJ as to be used in the present study and practiced it from all 3 experimental drop heights until they could demonstrate proficiency, which was subjectively judged by 2 trainers who had taught DJ training for a minimum of 5 years. The DJ was deemed proficient provided there was: (a) no significant horizontal travel between takeoff and landing; (b) a short duration in the landing phase; and (c) a toe-first landing pattern. Participants wore their own, appropriate athletic footwear during the test. Participants were instructed to refrain from eating for 2 hours before testing and from strenuous physical activity for 48 hours before testing.

\section{Procedures}

The pre-experiment warmup consisted of running on a treadmill at $6 \mathrm{mph}$ for 4 minutes followed by stretching exercises for the major muscles of the lower extremities (22). Each participant performed blocks of 3 DJs from 15, 30, and $45 \mathrm{~cm}$ with approximately 10 seconds recovery between each DJ from a given height. The order of drop jump heights was counterbalanced. In addition, in the nonfatigued condition, a further 2 minutes' rest was given between each block of DJs.

Each participant was instructed to jump vertically with maximum effort while trying to spend as little time on the floor as possible (23). The flooring was a $1.9-\mathrm{cm}$ polyurethane multipurpose covering laid over a cement surface. Data on impact acceleration, knee kinematics, and jump height were collected for each jump. The average of each block of 3 DJs was used in the subsequent analysis.

An incremental running protocol on a treadmill was used to induce whole body endurance fatigue (22). Participants began running at $6 \mathrm{mph}$ with a $3 \%$ grade with a $1.5 \%$ incremental increase in gradient applied every minute thereafter. A rating of perceived exertion (RPE) was taken every 2 minutes and used to determine when physiological fatigue had occurred. RPE was used because it is less invasive than more direct measures (eg, blood lactate concentration, oxygen uptake), it has been shown to be a physiologically valid tool for prescribing exercise intensity (33), and it has been used widely in this manner (14,22). An RPE of 17 ("very hard") was chosen to indicate when an endurance-based fatigued state was reached. Participants then repeated the DJs in the fatigued condition in the same order as carried out in the nonfatigued condition. The time from the end of the fatiguing protocol to when the DJ protocol was initiated again was always $<1$ minute. 


\section{Data Collection}

In reaction to the foot contacting the ground when drop jumping, an impact acceleration (or "shock wave") travels through the body, which, when excessive, may cause musculoskeletal injury (as detailed previously). Impact accelerations were measured at the proximal end of the tibia using a lightweight (17 g) uniaxial accelerometer (ADXL78; Analog Devices, Limerick, $\mathrm{UK}$; sensitivity $38 \mathrm{mV} / \mathrm{g}$, range $\pm 50 \mathrm{~g}$ ) attached to the skin overlying the proximal anteriormedial aspect of the right tibia. The accelerometer was attached to the skin with double-sided tape and prewrap (Durapore; 3M, Bracknell, UK) and aligned along the longitudinal aspect of the tibia. An elastic belt wrapped around the shank pressed the accelerometer onto the skin as tightly as comfortably allowed (35). It is recognized by the authors that accelerometers are sensitive to their orientation relative to gravity and in the present study were attached to the skin rather than directly on bone. Orientation sensitivity will introduce a nonconsistent error in measures of peak acceleration if either fatigue or drop height results in different tibia angles at landing. However, in a previous study on DJs from $30 \mathrm{~cm}$, this error was shown to be very small (1.5\%) (22). This potential error was taken into consideration when examining the magnitude of any effects in the present study.

Although mounting an accelerometer on the skin with double-sided tape may underestimate the magnitude of accelerations (12), the effect is considered to be relatively consistent across conditions (fatigued and nonfatigued) and, most importantly, does not require an invasive procedure. Skin-mounted accelerometers have been used previously to examine impact accelerations in running $(5,21,35)$ and DJs $(22)$.

To determine if changes in knee kinematics occurred after the fatiguing protocol, and if these changes may have explained any corresponding changes in the magnitude of impact acceleration, sagittal plane knee kinematics were examined. Knee angles were used to assess the angle of the knees at initial impact and takeoff and subsequently to determine the range of joint motion used. Knee angular velocity was used to assess the degree of knee control during the landing phase. Knee motion was measured using an electrogoniometer (XM110; Biometrics Ltd, Cwmfelinfach,UK) attached using doublesided tape and prewrap (Durapore) and aligned with the center of the knee on the lateral side of the left knee. The left knee was used in case the electrogoniometer made contact with the accelerometer and caused erroneous results. The electrogoniometer was zeroed before both the nonfatigued and the fatigued series of DJs as the subject stood upright with a straight leg. Knee angle data were smoothed using a fourth-order zero-lag Butterworth digital filter (37) with a cutoff frequency of $20 \mathrm{~Hz}$ to retain the subtleties in the velocity-time traces (22). Angular velocities were calculated by simple differentiation (37). The start and end of foot-to ground contact was registered using a foot switch apparatus (TF100; Biometrics Ltd) attached to the underside of the forefoot of each participant's right shoe.

The foot switch data were used to identify initial ground contact as well as the flight time of the subsequent jump, the latter of which was used to determine jump height as an indicator of neuromuscular fatigue. Jump height was calculated from flight time (3). To reduce the potential for damage to the switch, it was removed before the fatiguing running protocol; its site of attachment was clearly marked to facilitate accurate reattachment for the second set of DJs. The foot switch was tested before and after each experiment by pinching the pressure sensor and was found to work effectively on all occasions. The accelerometer, electro- 
goniometer and the switch were sampled simultaneously at a rate of $1,000 \mathrm{~Hz}$ (DataLINK; Biometrics Ltd).

To assess the reliability of the results, 8 participants repeated the test protocol on a second day using only the drop height of $30 \mathrm{~cm}$. A break of at least 4 days was given between test sessions.

\section{Statistical Analyses}

The average of each block of 3 trials was used in the analysis, a 2 (condition: nonfatigued and fatigued) by 3 (drop height: 15, 30, and 45m) within-subjects analysis of variance. Tibial peak impact acceleration, angle of knee flexion at ground contact, peak knee flexion during the landing phase, range of knee flexion between these 2 points, peak knee flexion angular velocity during the landing phase, and jump height were the dependent variables assessed. Where a significant main effect of drop height was found, a repeatedmeasures analysis of variance was performed followed by post hoc multiple paired t-tests with appropriate Bonferroni adjustment. Where a statistically significant interaction was found, planned comparisons using paired t-tests with appropriate Bonferroni adjustment were completed. The interday reliability of all dependent variables was assessed using intraclass correlation analysis (ICC). The level of statistical significance in all tests was set at $\rho=0.05$. SPSS for Windows version 10 (SPSS Inc, Chicago, IL) was used

\section{RESULTS}

Subjects ran on average for 8.6_+2.7 minutes, attaining a final gradient of $15.9++3.8 \%$ during the fatiguing protocol.The interday reliability (ICC) of measures ranged from 0.82 to 0.98 (Table 1).Tibial peak impact acceleration, angle of knee flexion at ground contact, peak knee flexion during the landing phase, range of knee flexion between these 2 points, peak knee flexion angular velocity during the landing phase, and jump height were the dependent variables assessed.

TABLE 1. Interday reliability (intraclass correlation) of test measures.

\begin{tabular}{lcc}
\hline & Fatigued & Nonfatigued \\
\hline Impact acceleration & 0.82 & 0.83 \\
Knee angle at ground contact & 0.92 & 0.93 \\
Peak knee flexion & 0.89 & 0.90 \\
Range of knee flexion & 0.95 & 0.94 \\
Peak knee flexion angular velocity & 0.93 & 0.93 \\
Jump height & 0.98 & 0.97 \\
\hline
\end{tabular}

\section{Tibial Peak Impact Acceleration}

Condition $(\mathrm{F}=36.8, \rho=0.000)$, drop height $(\mathrm{F}=103.34, \rho=0.000)$, and their interaction $(\mathrm{F}=$ $8.3, \rho=0.001$ ) had a significant effect on tibial peak impact acceleration (Figure 1). Post hoc analyses revealed that endurance fatigue had a significant effect at $15 \mathrm{~cm}(\mathrm{t}=-8.08, \rho=$ $0.000)$ and $30 \mathrm{~cm}(\mathrm{t}=-3.79, \rho=-.002)$, but not at $45 \mathrm{~cm}(\mathrm{t}=-1.21, \rho=0.25)$, with increases in peak accelerations of 32,17 , and $2 \%$, respectively. With each increase in drop height, peak impact acceleration also significantly increased in both fatigued $(\mathrm{F}=65.5, \rho=0.000)$ and nonfatigued $(\mathrm{F}=135.8, \rho=0.000)$ conditions (fatigued: 15 vs. $30 \mathrm{~cm}=37 \%[\mathrm{t}=-11.3, \rho=$ 
$0.000], 15$ vs. $45 \mathrm{~cm}=103 \%$ [ $\mathrm{t}=-14.0, \rho=0.000]$, and 30 vs. $45 \mathrm{~cm}=48 \%[\mathrm{t}=-8.9, \rho=$ $0.000]$; nonfatigued: 15 vs. $30 \mathrm{~cm}=55 \%[\mathrm{t}=-7.0, \rho=0.000], 15$ vs. $45 \mathrm{~cm}=162 \%[\mathrm{t}=-9.3, \rho$ $=0.000]$, and 30 vs. $45 \mathrm{~cm}=68 \%[\mathrm{t}=-6.6, \rho=0.000])$.

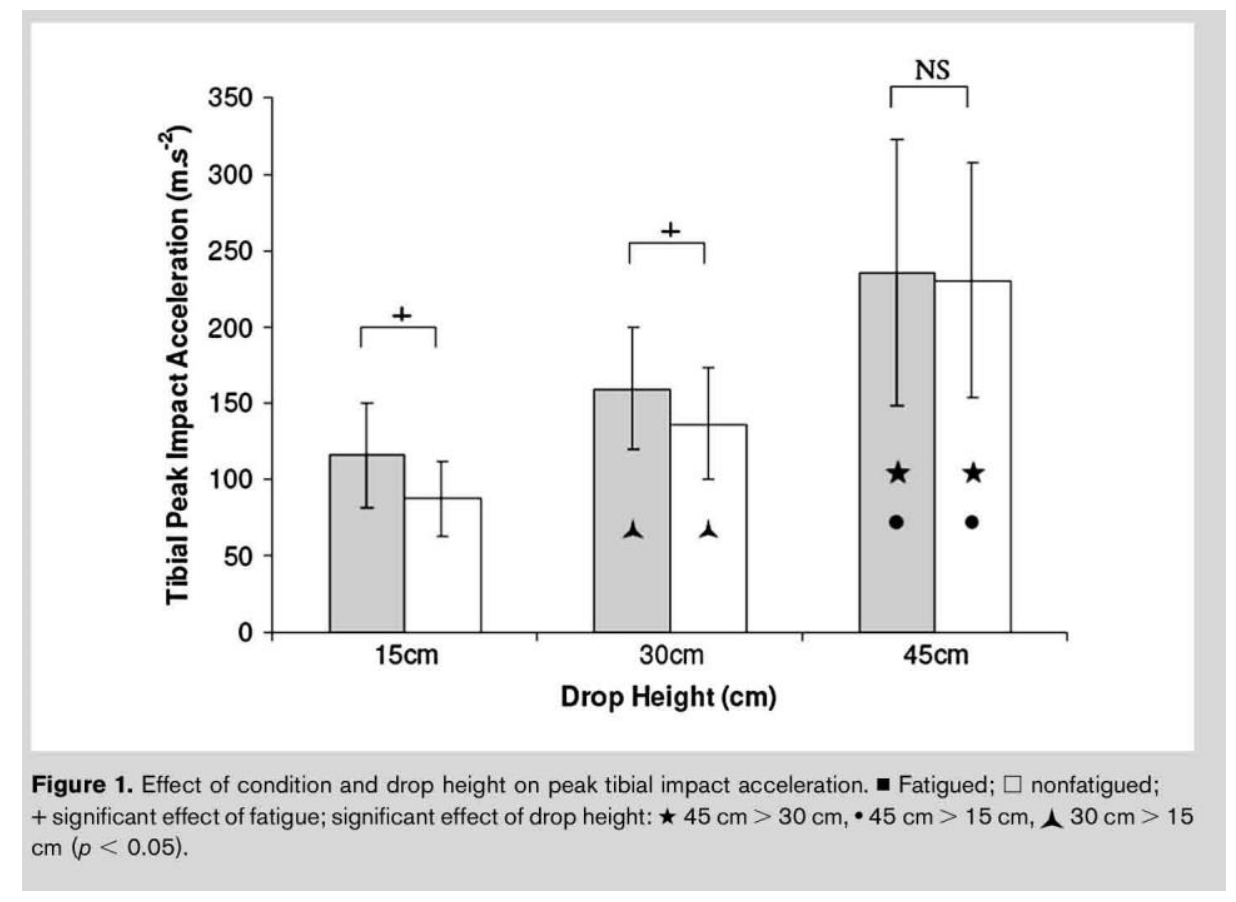

\section{Knee Joint Kinematics}

Only drop height had a significant effect on the angle of knee flexion at initial ground contact $(\mathrm{F}=194.8, \rho=0.000)$ with a significantly greater angle evident from $15 \mathrm{~cm}(23.8++5.2)$ and $30 \mathrm{~cm}(23.7+5.8)$ than from $45 \mathrm{~cm}\left(18.5+4.5 \_\right)$(Figure 2). Condition $(\mathrm{F}=1.05, \rho=0.32)$, drop height $(\mathrm{F}=1.65, \rho=0.21)$, and their interaction $(\mathrm{F}=0.72, \rho=0.49)$ did not have a significant effect on the angle of peak knee flexion (Figure 3). Only drop height significantly affected the range of knee flexion $(\mathrm{F}=7.0, \rho=0.003)$ with a greater range from $45 \mathrm{~cm}$ $(58.7++15.6)$ than from $30 \mathrm{~cm}(48.7++7.1)$ and $15 \mathrm{~cm}(48.3+9.9)$ (Figure 4). The same trend in results was evident for knee peak angular velocity during the eccentric phase as for tibial peak impact acceleration. Condition $(\mathrm{F}=190.5, \rho=0.000)$, drop height $(\mathrm{F}=54.5, \rho=0.000)$, and their interaction $(\mathrm{F}=27.1, \rho=0.000)$ were all significant (Figure 5). Post hoc analysis revealed that fatigue had a significant effect at $15 \mathrm{~cm}(\mathrm{t}=13.5, \rho=0.000)$ and $30 \mathrm{~cm}(\mathrm{t}=$ 14.3, $\rho=0.000)$, but not at $45 \mathrm{~cm}(\mathrm{t}=1.6, \rho=0.13)$ with increases of 21,20 , and $2 \%$, respectively. Increased drop height also had a significant affect when both fatigued $(\mathrm{F}=62.9$, $\rho=0.000)$ and nonfatigued ( $\mathrm{F}=42.2, \rho=0.000)$ with increases in angular velocity evident at each increase in drop height (fatigued: 15 vs. $30 \mathrm{~cm}=24 \%[\mathrm{t}=-4.9, \mathrm{p}=0.000], 15$ vs. $45 \mathrm{~cm}$ $=72 \% \quad[\mathrm{t}=26.5, \rho=0.000]$, and 30 vs. $45 \mathrm{~cm}=40 \%[\mathrm{t}=26.5, \rho=0.000]$; nonfatigued: 15 vs. $30 \mathrm{~cm}=24 \%[\mathrm{t}=24.2, \rho=0.001], 15$ vs. $45 \mathrm{~cm}=104 \%[\mathrm{t}=26.5 \rho=0.000]$, and $30 \mathrm{vs}$. $45 \mathrm{~cm}=64 \%(\mathrm{t}=26.5, \rho=0.000])$ 


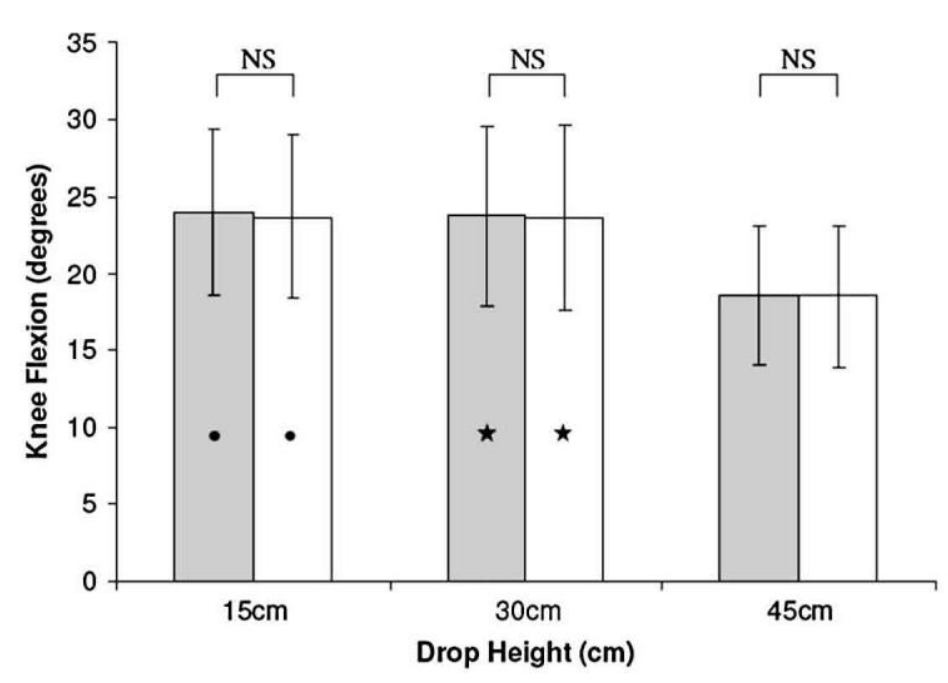


$\square$ nonfatigued; + significant effect of fatigue; significant effect of drop height: $\star 30 \mathrm{~cm}>45 \mathrm{~cm}, \bullet 15 \mathrm{~cm}>45 \mathrm{~cm}$ $(p<0.05)$.

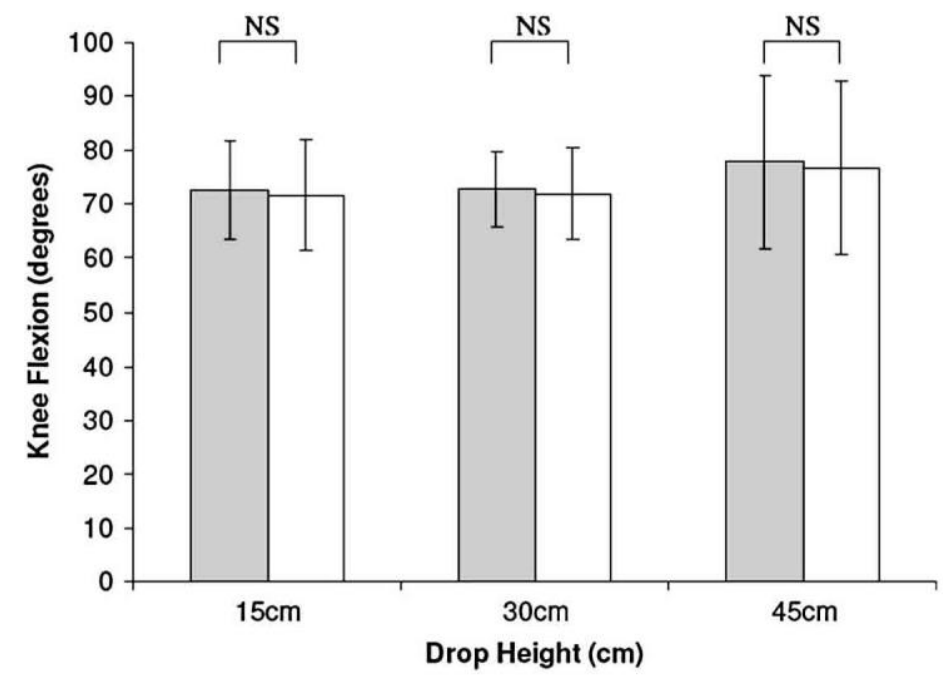

Figure 3. Effect of condition and drop height on angle of peak knee flexion. $₫$ Fatigued; $\square$ nonfatigued; + significant effect of fatigue; $\star$ significant effect of drop height $(p<0.05)$. 




Figure 4. Effect of condition and drop height on range of knee flexion. $\square$ Fatigued; $\square$ nonfatigued; + significan effect of fatigue; significant effect of drop height: $\star 45 \mathrm{~cm}>30 \mathrm{~cm}, \bullet 45 \mathrm{~cm}>15 \mathrm{~cm}(p<0.05)$.

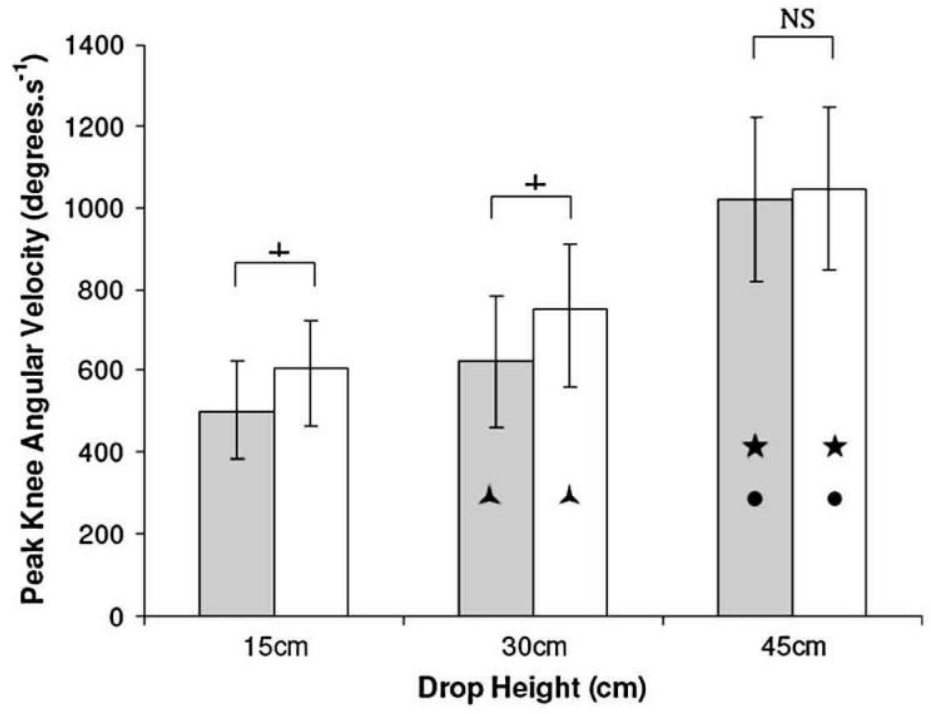

Figure 5. Effect of condition and drop height on knee joint peak angular velocity. $\approx$ Fatigued; $\square$ nonfatigued; + significant effect of fatigue; significant effect of drop height: $\star 45 \mathrm{~cm}>30 \mathrm{~cm}, \bullet 45 \mathrm{~cm}>15 \mathrm{~cm}, \lambda 30 \mathrm{~cm}$ $>15 \mathrm{~cm}(p<0.05)$.

\section{Jump Height}

Both condition $(\mathrm{F}=187.8, \rho=0.000)$ and drop height $(\mathrm{F}=24.9, \rho=0.000)$ had a significant effect on jump height (Figure 6). Post hoc analyses revealed that jump height was greater from both 45 and $30 \mathrm{~cm}$ than from $15 \mathrm{~cm}$. Endurance fatigue caused a similar reduction in jump height at all 3 drop heights (approximately $8 \%$ ). 


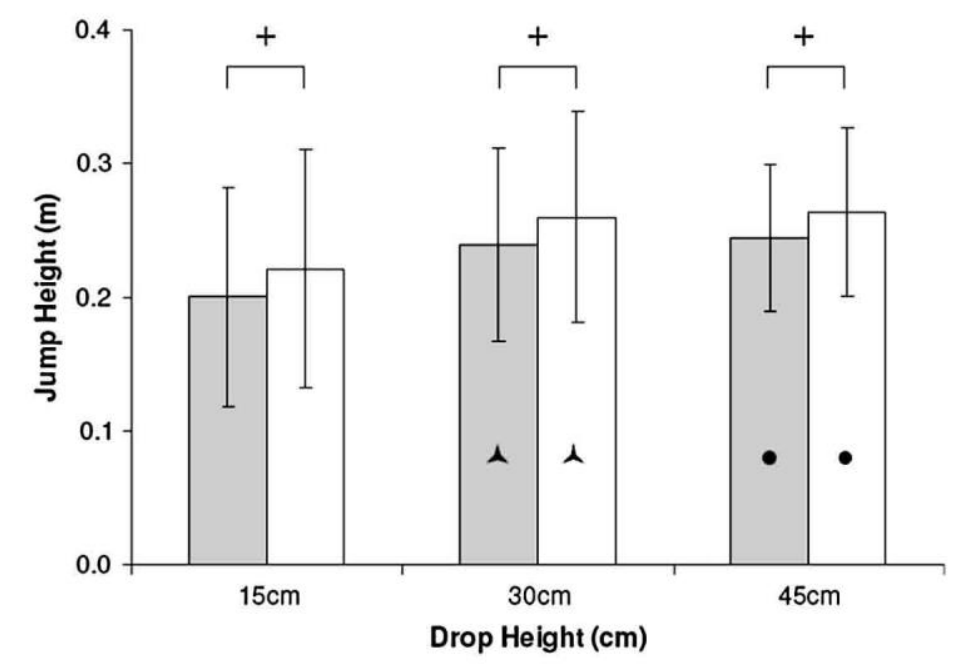

Figure 6. Effect of condition and drop height on jump height. $\square$ Fatigued; $\square$ nonfatigued; + significant effect of fatigue; significant effect of drop height: $\bullet 45 \mathrm{~cm}>15 \mathrm{~cm}, \lambda 30 \mathrm{~cm}>15 \mathrm{~cm}(p<0.05)$.

\section{DISCUSSION}

Based on the decrease in jump height after the fatiguing running protocol (Figure 6), endurance-based neuromuscular fatigue appears to have been induced, indicating the appropriate use of the running protocol and the PRE scale. Although not assessed directly in the present study, it is likely that both central (neural) and peripheral (contractile component) activation pathways were affected (24).

Although it is generally recommended that DJs are performed when the neuromuscular system is not fatigued (31), thereby maximizing neural activation, the principle of specificity clearly indicates that for athletes who need to produce high-power output when fatigued, there is a need to develop lower limb power under such fatigued conditions (7). DJ training is commonly used to increase lower limb power. However, this gives rise to the question of whether completing DJs when fatigued will result in an increase in impact accelerations, which are associated with an increase in the risk of injury $(6,16,26)$. The present study found a significant interaction between endurance fatigue and drop height for impact accelerations measured at the proximal tibia during drop jumping. Tibial accelerations were significantly larger from drop heights of $15 \mathrm{~cm} \mathrm{(32 \% )} \mathrm{and} 30 \mathrm{~cm}$ (17\%) when participants were fatigued in comparison to nonfatigued, but no significant difference was evident from the higher drop height of $45 \mathrm{~cm}(2 \%)$ (Figure 1). In addition, the increase in peak acceleration associated with endurance fatigue was significantly larger from 15 than $30 \mathrm{~cm}$.

This trend in results showing a negative effect of fatigue, especially at lower drop heights, is in agreement with the hypothesis of the present study and the findings of Moran and Marshall (22).The findings are interpreted as indicating that at greater heights, the neuromuscular system's capacity to control the greater impact acceleration per se is notably reduced irrespective of whether it is fatigued or nonfatigued. Support for this assertion is taken from 2 sources. First, in the present study, a similar pattern of results was found for the effect of drop height and endurance fatigue on knee flexion peak angular velocities during the landing phase as that for impact accelerations (Figures 1 and 5). Because the neuromuscular system 
acts to decrease knee angular velocities during the landing phase, the knee angular velocity data illustrate that the neuromuscular system had a reduced capacity during the loading phase in DJs from $45 \mathrm{~cm}$, whether fatigued or nonfatigued, but was able to positively affect jumps from 15 and $30 \mathrm{~cm}$. Second, in comparing findings from the present study with 2 previous studies on running $(20,35)$ there is a clear trend that as impact accelerations increase per se, the relative effect of fatigue diminishes. For running, in which impact accelerations are lower than DJs (approximately $68 \mathrm{~m} \cdot{ }^{\mathrm{S}-2}$ [20]), Mizrahi et al (20) and Voloshin et al (35) reported that endurance fatigue caused an increase in proximal tibial peak acceleration of $62 \%$ and approximately $60 \%$, respectively. In contrast, in the present study, as impact accelerations increased with increases in drop height (on average: $102.0 \pm 24.3 \mathrm{~m} . \mathrm{S}^{-2}$ from $15 \mathrm{~cm}, 148.1 \pm$ $27.4 \mathrm{~m} . \mathrm{S}^{-2}$ from $30 \mathrm{~cm}$, and $232.8 \pm 58.9 \mathrm{~m} . \mathrm{S}^{-2}$ from $45 \mathrm{~cm}$ ), the relative effect of endurance fatigue decreased ( $32 \%$ from $15 \mathrm{~cm}, 17 \%$ from $30 \mathrm{~cm}$, and just $2 \%$ from $45 \mathrm{~cm}$ ). Although the present study did not attempt to identify the individual neuromuscular mechanisms responsible for attenuating impact accelerations, it is worth noting that endurance fatigue causes a decrease in proprioception $(10,19)$ and contractile and reflex capacities (9) in stretch shortening activities such as drop jumping, which are important mechanisms in controlling the eccentric loading phase (9). Previous studies on the affect of endurance fatigue in running have suggested that changes in tibial peak impact accelerations may be the result of changes in the angle of knee flexion at key times during the landing phase $(5,21)$. The present study examined the effect of fatigue on the angle of knee flexion at initial ground contact, the end of the eccentric phase, and the range of motion between them. Fatigue was found to have no effect on these kinematic measures. Similar results have been reported previously (17), although these authors did note an increase in the angle of knee abduction and internal rotation. The contrast in results between DJs and running may be the result of the task requirement in DJs to remain in contact with the ground for as short a duration as possible or to the explosive action being less practiced than running, and the coordination pattern therefore being less susceptible to relative change, even when the neuromuscular system's capacity is compromised when fatigued (28). Therefore, the findings from the present study indicate that changes in peak impact accelerations induced by endurance fatigue cannot be explained by changes in the knee angles at key points during the landing phase. Although not assessed in the present study, it is possible that changes in hip or ankle kinematics may have occurred. However, in examining the effect of endurance fatigue on drop jumps from $50 \mathrm{~cm}$, McClean et al found no changes in ankle and hip (dorsi-) flexion, abduction, or internal rotation.

\section{Limitations to the Present Study}

This study is based on the premise that a significant increase in impact accelerations will increase the risk of injury, yet there is a dearth of information available on what constitutes an "excessive" impact acceleration or load. In referring mainly to animal model studies that directly found joint cartilage and bone degeneration after high impact loading $(6,27,36)$ and also to epidemiologic studies on subjects exposed to high loadings during their chosen activity (18), the general litera-ture simply refers to "excessive" impact accelerations and/or loads predisposing to injury $(13,16,18,21,26)$. Although a plyometric DJ training session may 
contain only between 30 and 100 ground impacts, tissue damage appears to be more dependent on the magnitude of loading rather than the number of impacts (36).

\section{PRACTICAL APPLICATIONS}

Given that the principle of "training specificity" indicates that to maximize power output during fatiguing conditions (eg, end of a basketball match), it may be beneficial to train power when fatigued, cognizance should be taken of any potential increase in the likelihood of injury associated with completing such exercises when fatigued. Although data do not currently exist on what constitutes an "excessive" magnitude of impact acceleration during DJ training, in terms of predisposing an individual to a greater risk of injury (eg, stress fractures, articular cartilage and joint degeneration, and osteoarthritis) $(6,16,26)$, the present study indicates that caution should be taken in deciding to undertake plyometric DJs $(15,30$, and $45 \mathrm{~cm}$ ) when fatigued because of an associated significant increase in impact accelerations. However, in interpreting the findings of the present study, it is important to note that smaller impact accelerations were evident when performing DJs from $15 \mathrm{~cm}$ when fatigued than from 30 and $45 \mathrm{~cm}$ when nonfatigued. Similarly, smaller impact accelerations were evident when performing DJs from $30 \mathrm{~cm}$ when fatigued than from $45 \mathrm{~cm}$ when nonfatigued (Figure 1). This implies that it may still be safer, or at least as safe, to DJ from an appropriately low(er) drop height when fatigued than from a high(er) drop height when nonfatigued.

\section{REFERENCES}

1. Bobbert, MF. Drop jumping as a training method for jumping ability. Sports Med 9: 7-22, 1990. 2. Bobbert, MF, MacKay, M, Schinkelshoek, D, Huijing, PA, and Van Ingen Schenau, GJ. Biomechanical analysis of drop and counter-movement jumps. Eur J Appl Physiol 54: 566-573, 1986.

3. Bosco, C and Komi, PV. Mechanical characteristics and fibre composition of human leg extensor muscles. Eur J Appl Physiol 41: 275-284, 1979.

4. Derrick, TR. The effects of knee contact angle on impact forces and accelerations. Med Sci Sports Exerc 36: 832-837, 2004.

5. Derrick, TR, Dereu, D, and McLean, SP. Impacts and kinematic adjustments during an exhaustive run. Med Sci Sports Exerc 34: 998-1002, 2002.

6. Ewers, BJ, Dvoracek-Driksna, D, Orth, MW, and Haut, RC. The extent of matrix damage and chondrocyte death in mechanically traumatized articular cartilage explants depends on the rate of loading. J Orthop Res 19: 779-784, 2001.

7. Fleck, SJ and Kraemer, WJ. Designing Resistance Training Programs. Leeds, UK: Human Kinetics, 2004.

8. Flynn, JM, Holmes, JD, and Andrews, DM. The effect of localized leg muscle fatigue on tibial impact acceleration. Clin Biomech 19: 726-732, 2004.

9. Gollhofer, A, Komi, PV, Fujitsuka, N, and Miyashita, M. Fatigue during stretch-shortening cycle exercises. II. Changes in neuro-muscular activation patterns of human skeletal muscle. Int J Sports Med 8: 38-41, 1987.

10. Hiemstra, LA, Lo, IK, and Fowler, PJ. Effect of fatigue on knee proprioception: implications for dynamic stabilization. J Orthop Sports Phys Ther 31: 598-605, 2001. 
11. Komi, P and Bosco, C. Utilisation of stored elastic energy in leg extensor muscles by men and women. Med Sci Sports Exerc 10: 261-265, 1978.

12. LaFortune, MA, Hennig, EM, and Valiant, GA. Tibial shock measured with bone and skin mounted transducers. J Biomech 28: 989-993, 1995.

13. LaFortune, MA, Lake, MJ, and Hennig, EM. Differential shock transmission response of the human body to impact severity and lower limb posture. J Biomech 29: 1531-1537, 1996.

14. Lamb, K, Eston, R, and Corns, D. Reliability of ratings of perceived exertion during progressive treadmill exercise. Br J Sports Med 33: 336-339, 1999.

15. Light, LH, McLellan, GE, and Klenerman, L. Skeletal transients on heel strike in normal walking with different footwear. J Biomech 13: 477-480, 1980.

16. Madigan, ML and Piecoe, PE. Changes in landing biomechanics during a fatiguing landing activity. J Electromyogr Kinesiol 13: 491-498, 2003.

17. McLean, SG, Felin, RE, Suedekum, N, Calabrese, G, Passerallo, A, and Joy, S. Impact of fatigue on gender-based high-risk landing strategies. Med Sci Sports Exerc 39: 502-514, 2007.

18. Milgrom, C. The Israeli elite infantry recruit: a model for understanding the biomechanics of stress fractures. J R Coll Surg Edinb 34: 18-21, 1989.

19. Miura, K, Ishibashi, Y, Tsuda, E, Okamura, Y, Otsuka, H, and Toh, S. The effect of local and general fatigue on knee proprioception. J Arthrosc Relat Surg-

20: 414-418, 2004. 20. Mizrahi, J, Verbitsky, 0, and Isakov, E. Shock accelerations and attenuation in downhill and level running. Clin Biomech 15: 15-20, 2000.

21. Mizrahi, J, Verbitsky, 0, Isakov, E, and Daily, D. Effect of fatigue on leg kinematics and impact acceleration in long distance running. Hum Mov Sci 19: 139-151, 2000.

22. Moran, KA and Marshall, BM. Effect of fatigue on tibial impact accelerations and knee kinematics in drop jumps. Med Sci Sports Exerc 38: 1836-1842, 2006.

23. Newton, R and Dugan, E. Application of strength diagnosis. Strength and Conditioning Journal 24: 50-59, 2002. 24. Noakes, T. Physiological models to understand exercise fatigue and the adaptations that predict or enhance athletic performance. Scand J Med SC7. Sports 10: 123-145, 2000.

25. Radin, EL. Role of muscle in protecting athletes from injury. Acta Media Scandica 711: 143-147, 1986.

26. Radin, EL, Eyre, D, Kellman, JL, and Schiller, AL. Effect of prolonged walking on concrete on the joints of sheep. Arthritis Rheum 22: 649, 1980.

27. Radin, EL, Parker, HG, Pugh, SW, Steinberg, RS, Paul, IL, and Rose, RM. Response of joints to impact loading-III: Relationship between trabecular microfractures and cartilage degeneration. J Biomech 6: 51-57, 1973.

28. Rodacki, AL, Fowler, NE, and Bennett, SJ. Multi-segment co-ordination: Fatigue effects. Med Sci Sports Exerc33: 1157-1167, 2001.

29. Saunders, P, Telford, R, Pyne, D, Pelyola, E, Cunningham, R, Gore, C, and Hawley, J. Short-term plyometric training improves running economy in highly trained middle distance and long distance runners. Journal of Strength and Conditioning Research 20: 947-954, 2006. 30. Schmidtbleicher, D. Training for power events. In: Strength and Power in Sport. Encyclopaedia ofSports Medicine. PV Komi, ed. Oxford, UK: Blackwell Science, 1992. pp. 381-395. 
31. Siff, MC and Verkhoshansky, YV. Supertraining. Denver, CO: Supertraining International 1999.

32. Spurrs, RW, Murphy, AJ, and Watsford, ML. effect of plyometric training on distance running and performance. Eur J Appl Physiol89: 1-7, 2003.

33. Steed, J, Gaesser, G, and Weltman, A. Rating of perceived exertion and blood lactate concentration during submaximal running. Med Sci Sports Exerc 26: 797-803, 1994.

34. Turner, AM, Owings, M, and Schwane, JA. Improvement in running economy after 6 weeks of plyometric training. Journal of Strength and Conditioning Research 17: 60-67, 2003.

35. Voloshin, AS, Mizrahi, J, Verbitsky, 0, and Isakov, E. Dynamic loading on the human musculoskeletal system-Effect of fatigue. Clin Biomech 13: 515-520, 1998.

36. Whiteside, RA, Jakob, RP, Wyss, UP, and Mainil-Varlet, P. Impact loading of articular cartilage during transplantation of osteochondral autograft. J Bone Joint Surg Br 87: 12851291, 2005.

37. Winter, DA. Biomechanics and Motor Control of Human Movement. New York: WileyInterscience Publication, 2005.

38. Wosk, J and Voloshin, A. Wave attenuation in skeletons of young healthy persons. J Biomech 14: 261-267, 1981.

39. Yoshikawa, T, Mori, S, Santiesteban, AJ, Sun, TC, Hafstad, E, Chen, J, and Burr, DB. The effects of muscle fatigue on bone strain. J Exp Biol 188: 217-233, 1994.

40. Young, WB, Wilson, GJ, and Byrne, C. A comparison of drop jump training methods: Effects on leg extensor strength qualities and jumping performance. Int J Sports Med 20: 295-303, 1999. 